\title{
A gastrocnemius heterotopical transplant model with end-to-side neurorraphy ${ }^{1}$
}

\author{
Marcos Ricardo de Oliveira Jaeger ${ }^{\mathrm{I}}$, Jefferson Luis Braga Silva", James Bain ${ }^{\mathrm{III}}$, Pedro Bins Ely ${ }^{\mathrm{IV}}$, Jefferson André Pires ${ }^{\mathrm{V}}$, Lydia \\ Masako Ferreira ${ }^{\text {II }}$
}

DOI: http://dx.doi.org/10.1590/S0102-8650201400140010

IFellow Postdoc degree, Postgraduate Program in Plastic Surgery, Paulista School of Medicine, Federal University of São Paulo (UNIFESP-EPM), Brazil. Instructor of the Departament of Plastic surgery, Santa Casa of Porto Alegre, Brazil. Conception, design, intellectual and scientific content of the study.

${ }^{I I} \mathrm{PhD}$, Associate Professor, Hand Surgery and Reconstructive Microsurgery Departament, Hospital São Lucas, Pontifícia Universidade Católica do Rio Grande do Sul (PUC-RS), Brazil. Conception and design of the study, critical revision.

IIIPhD, Full Professor, Division of Plastic Surgery, McMaster University, Canada. Intellectual and scientific content of the study, critical revision.

${ }^{\mathrm{IV}} \mathrm{PhD}$, Associate Professor, Division of Plastic Surgery, Federal University of Health Science, Surgeon-in-chief of Division of Plastic Surgery, Santa Casa of the Porto Alegre, Brazil. Manuscript writing, critical revision.

${ }^{\vee}$ MD. Resident, Departament of Surgery, Federal University of Pelotas, Brazil. Member of Brazilian College of Surgeons, Brazil. Manuscript writing, critical revision.

${ }^{{ }^{V}}$ Head and Full Professor, Plastic Surgery Division, UNIFESP, Researcher 1A-CNPq, Director Medicine III-CAPES, Sao Paulo-SP, Brazil. Manuscript writing, critical revision.

\section{ABSTRACT}

PURPOSE: To present an animal model to assess the effects of end-to-side innervation in the heterotopically transplanted model with reduced chances of neural contamination.

METHODS: The medial portion of the gastrocnemius muscle in wistar male rats was isolated and its pedicle dissected and performed a flap in the abdominal portion. To prevent neural contamination in the abdominal region, the muscle was wrapped with a Goretex ${ }^{\circledR}$ sheet. The specimens were divided into 2 groups (G). In G1 was performed an end-to-end suture between tibial nerve of the gastrocnemius and femoral motor nerve and between the saphenous sensory nerve and the motor nerve. In G2 was performed a end-to-side suture between the tibial nerve and the motor femoral and between the tibial nerve and saphenous motor nerve. The specimens were evaluated 60 days later to check the structure of the neurorraphy. Sections were obtained proximal and distal to the coaptation site.

RESULTS: The medial gastrocnemius muscle had the advantage of maintaining visible mass after 60 days. No disruption of the coaptation site was found. No major injury to the donor nerve was seen in group 2.

CONCLUSION: The proposed model is simple, reproduciple and prevent the neural contamination in the flap in end-to-side suture. Key words: Animal model. Mycrosurgery. Rats. 


\section{Introduction}

Ballance reported the utility of an end-to-side neurorraphy for facial nerve reconstruction. Harris and Low reported the usage of the technique for brachial plexus reconstruction ${ }^{1,2}$. At that time, the procedure invariably resulted in some loss of function of the donor nerve. An attempt was then made to preserve the donor nerve by a non-injury approach through a longitudinal incision in the donor nerve sheath, exposing but not injuring the nerve fibers. The results were not satisfactory, with still some degree of loss maybe due to surgical trauma or inappropriate instruments.

Decades later, Viterbo ${ }^{3}$ reintroduced the concept using a transected peroneal nerve coapted to the side of the tibial nerve, without removal of the epineurial sheath. Then it was Lundborg $^{4}$ to use end-to-side neurorraphy utilizing a sensory and motor nerve. His model utilized a sciatic nerve donor uninjured except for a perineurial window. The contralateral sciatic nerve served as a trunk graft which was connected to the side of the sciatic nerve under study with no distal connectivity. The model avoided proximal injury, but suffered from a targetless nerve graft. Concerned about the proximal injury to the donor nerve, Noah developed an animal model utilizing a nerve graft arising end-toside from the sciatic nerve to the stump of the obturator nerve. The designed experimental model did not injure the donor nerve but utilized a nerve with predominant motor fibers. The gastrocnemius muscle in rats has been utilized previously as a transplant model. Its anatomy was understood in detail and assessed previously in reliability as a transplant model. This muscle has the advantage of bulk, functional characteristics, innervation and typical vascular supply ${ }^{6,7}$. The purpose of this paper is to present a simple transplant model to study the effects of the donor motor and a purely sensory nerve connected to the motor recipient nerve without the utilization of a nerve graft. The donor sensory nerve - the cutaneous saphenous nerve - is much smaller in caliber compared to the recipient nerve and is connected end-to-side, or rather, sideto-end to the recipient motor nerve. In the recipient region, the muscle is placed so that minimal interference from the surround tissues may occur.

\section{Methods}

Twelve Wistar male rats weighting 200-300g were utilized in the present study. The rules of the Brazilian National Research Council's guide for the utilization of laboratory animals were followed strictly. Anesthesia was obtained utilizing sodium pentobarbital in the dosage of $25 \mathrm{mg} / \mathrm{kg}$ given intraperitoneally.
The medial portion of the gastrocnemius muscle was isolated and its pedicle dissected further proximally to the origin at the level of the femoral vessels (average of 1 to $1.6 \mathrm{~mm}$ in diameter). The gastrocnemius motor nerve was then cut and the flap transposed to the abdominal region where it could be connected to the homolateral femoral vessels. The saphenous nerve was dissected and isolated (Figures 1,2).

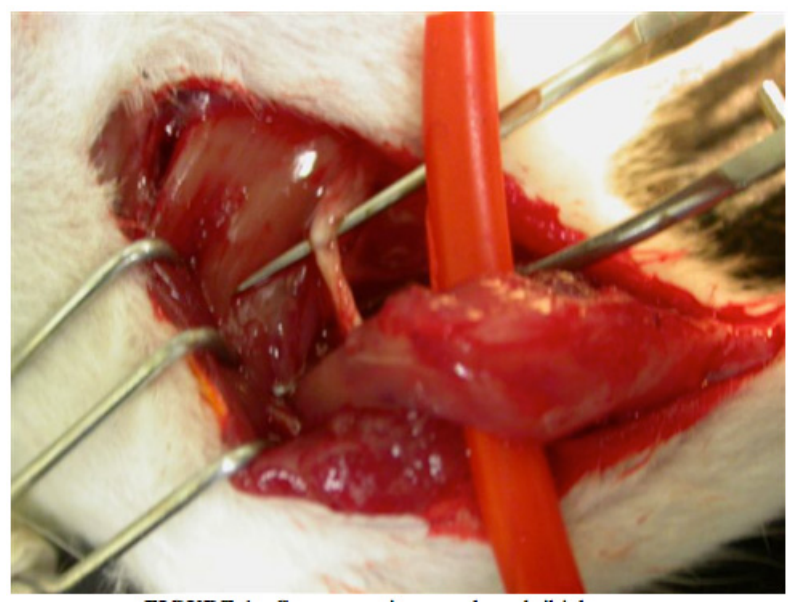

FIGURE 1 - Gastrocnemius muscle and tibial nerve.

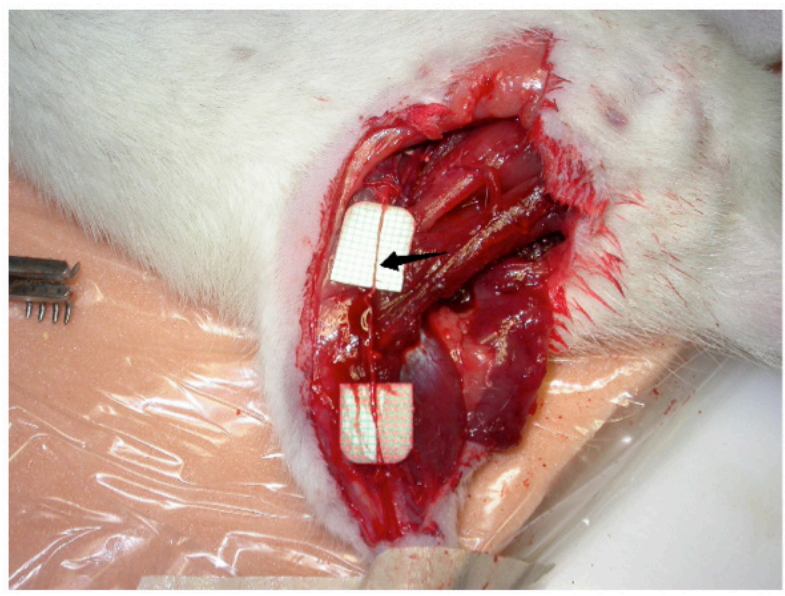

FIGURE 2 - Saphenous nerve.

To prevent neural contamination in the abdominal region, the muscle was wrapped with a $0.1 \mathrm{~mm}$ Goretex ${ }^{\circledR}$ sheet. A thick layer of adipofascial tissue can be easily identified at the abdominal region, and this flap may also be used to protect the alloplastic material against extrusion (Figure 3). The muscle was then securely attached to the abdominal fascia and, under light microscope; the motor nerve to the gastrocnemius received different treatments. Great great care was taken to preserve the function and structure of the donor nerve, especially the donor sensitive nerve, which was approximately half the diameter of the recipient motor nerve. In all specimens with end-to-side junction of the nerves, we created a very small perineurial window. The specimens were divided into 2 groups to check the constancy and anatomy of the model. In group 
1 (end-to-end sutures), the tibial nerve of the gastrocnemius muscle was sutured end-to-end to recipient femoral motor nerve, or the saphenous sensory nerve was connected end-to-end to the motor nerve. In group 2 (and-to-side sutures), the tibial nerve was cut and brought in length enough to reach end-to-side the motor femoral, or the tibial nerve was then brought in length to be connected end-toside to the saphenous sensitive nerve.

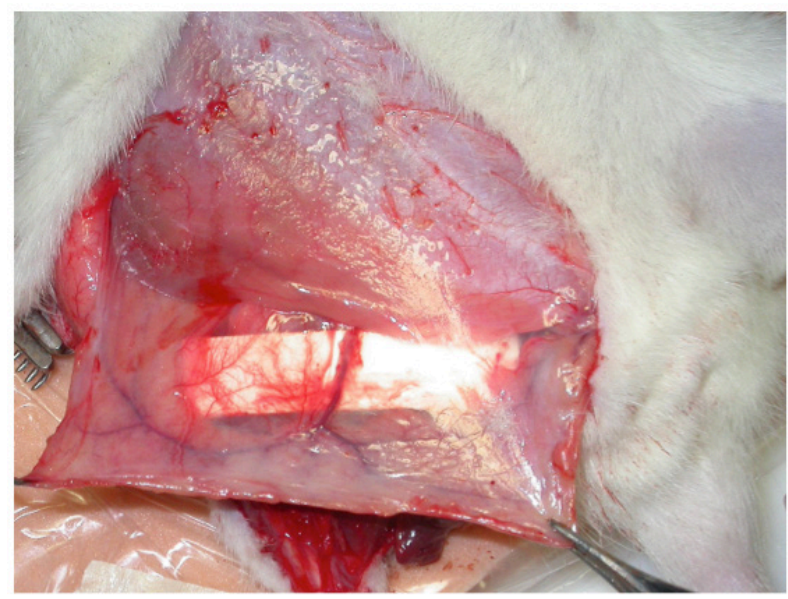

FIGURE 3 - Abdominal flap to protect the Goretex ${ }^{\circledR}$ sheet.

The specimens were evaluated 60 days later to check the structure of the neurorraphy. After post-fixation of the nerve specimens in 2 percent glutaraldehyde and 2 percent paraformaldehyde, the specimens were cut into two segments, and the nerve coaptation section verified for the presence of axonal sprouting. The coaptation site was cut in longitudinal sections and rinsed in cacodylate buffer for $20 \mathrm{~min}$, post-fixed in 1 percent osmium tetroxide for $2 \mathrm{hr}$ and embedded in epoxy resin Epon 812. Specimens were then stained in toluidine blue, cut with microtome into $1 \mu \mathrm{m}$ sections and examined under electron microscopy (Figures 4,5). Sections were obtained proximal and distal to the coaptation site.

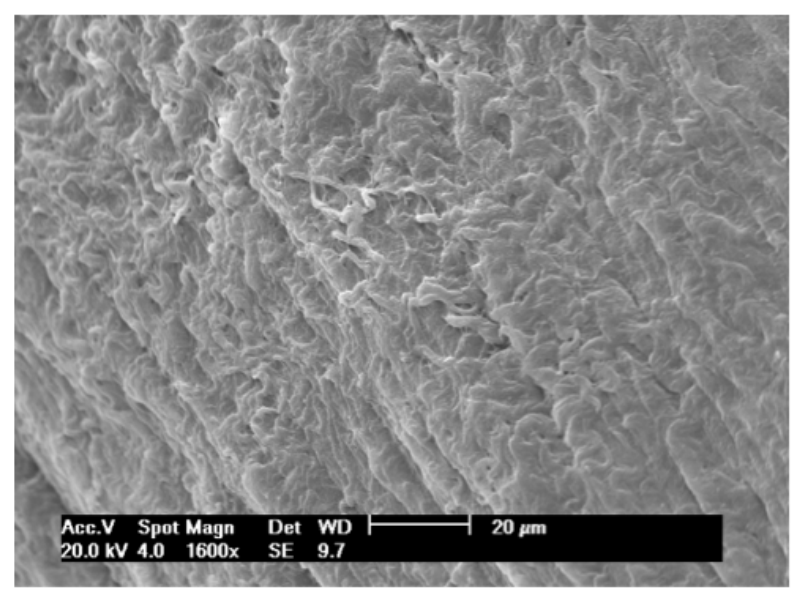

FIGURE 4 - Fiber alignment below perineurium.

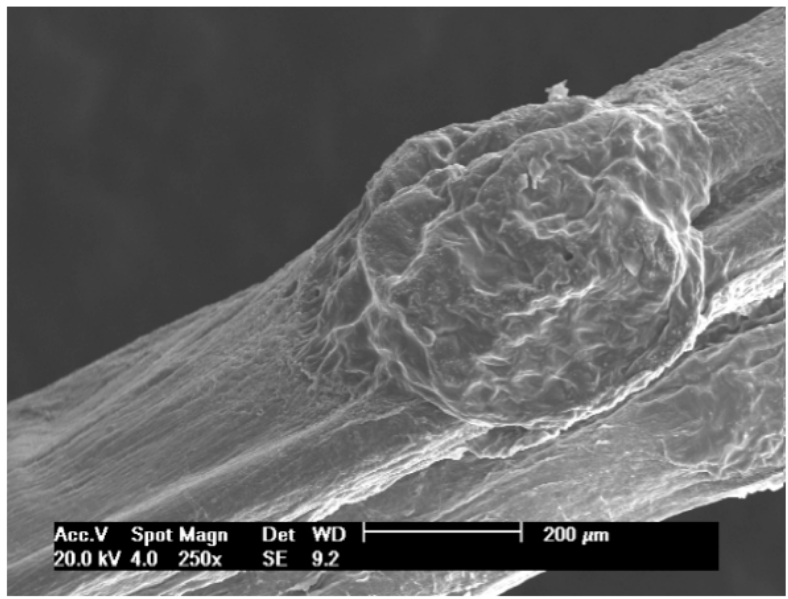

FIGURE 5 - Neuroma below perineurium.

\section{Results}

All animals survived the surgery and showed no major functional impairment, infection, or self-mutilation. The medial gastrocnemius muscle was found to have bulk, functional characteristics, innervation and distinctive vascular supply. The medial gastrocnemius had the advantage of maintaining visible mass after 60 days (Figure 6).

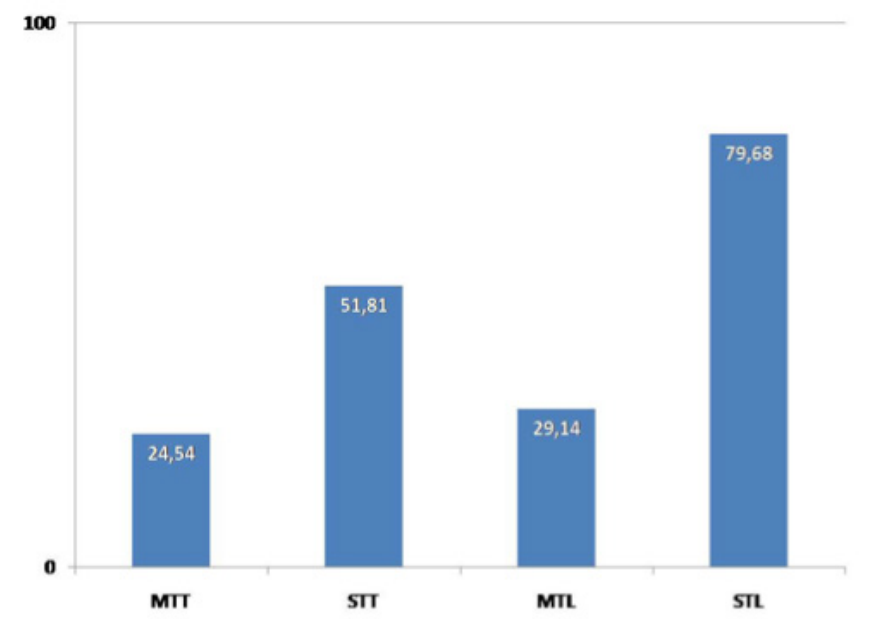

FIGURE 6 - Variation of wet weight of gastrocnemius muscle for suture tipe. MTT: end-to-end suture between motor nerves; MTL: end-to-side suture between motor nerves; STT: end-to-end suture between sensitive and motor nerves; STL: end-to-side suture between sensitive and motor nerves.

Regarding the nerve junction, no disruption of the coaptation site was found when longitudinal sections were examined in 3-day-old specimens. The coaptation of the two nerves appeared intact, and a close alignment of the perineurium of both nerves at the coaptation "T" junction was perceptible. Axonal sprouting was present in all end-to-side specimens.

No major injury to the donor nerve was seen. However, morphologic differences may be observed especially in the amount of regenerated axons and the density of the endoneurium in the group 2. 


\section{Discussion}

In the past, many experimental models have been utilized to study effects of denervation and reinnervation in the target organ/ nerve. The sensory nerve has proven to be beneficial to protect the disrupted motor nerve, maintaining receptor motor nerve function and structure ${ }^{8}$. To be clinically useful, the donor nerve should not be disrupted being still able to innervate its donor region. Endto-side neurorraphy could provide the answer. A regional sensory nerve could be utilized without disruption of its continuity towards the skin. Crucial is the way the nerve is brought to the end-to-side junction. The animal model utilizing an end-to-side neurorraphy with the peroneal nerve working as a graft and the posterior tibial nerve acting as the donor nerve had been the standard model for several subsequent reports. However, this model was criticized due to both focal demyelinization and a too large perineurial window. Other authors have utilized the end-to-side neurorraphy focusing on the functional reestablishment, which is better when two motor nerves are used instead of a sensory nerve. Tarasidis ${ }^{9}$ has shown that sensory regeneration occurs in all of the end-to-side groups, in contrast to motor regeneration that had shown to be present in a much smaller amount. The clinical impact of utilizing the sensory nerve end-to-side to the motor nerve may be one of two: maintain the structure of the motor nerve viable, and possibly keep the viability of the end-plate after disruption of the motor nerve.

Previous investigators have demonstrated axonal growing within a nerve graft independent of predenervation, even without removal of the envelope of connective tissue of the donor nerve on the repair site, truth contested by others ${ }^{5,8}$. In fact, removal of any connective tissue barrier, as well as a window of the perineurium, is strongly recommended, fact described by Noah as a way to promote the "small parasitic invasion of the graft by axons of unknown origin".

Several animal models have been utilized in the past. Many mechanisms could interfere in the evaluation of the results, and possibly pose obstacles in finding clinical applications. First, non-injury end-to-side models should still be able to inervate the initial target so that minimal morbidity would occur at the donor region. In the present model, motor nerves, and their targets, are kept the same. Second, direct co-aptation avoids the utilization of a nerve graft as a conduct for axonal sprouting across 2 repair sites. Third, often in the animal models the muscle is transplanted at the same original region - orthotopic, and very often tiny little lower limb muscles. In the present report, the medial gastrocnemius had the advantage of maintaining visible mass after 60 days, in spite of the fact that this was not an aim of the present study. This model can therefore be utilized to study the effects of atrophy in the future.
And finally, most of the criticism of the animal models arises from the fact that there might be the possibility of graft invasion by axons from the harvesting point. The animal model to study the variables of sensory input and end-to-side neurorraphy should include effective measures to lower the risk of neural contribution other than the desired effect of the sensory nerve ${ }^{9}$. We utilized a sheet of aloplastic material to prevent the graft, and the nerve junction, against ectopic reinnervation.

Axonal growth within a nerve graft at 30 days postoperatively has already been demonstrated in the literature ${ }^{4,5}$ and it was followed by the maintenance of the wet weight of the target muscle. The injury itself may have worked to stimulate exaggerated regeneration. The key point is to show at the nerve coaptation site that there is not only axonal growing, but axonal sprouting from the donor nerve connected. Axonal sprouting represents the way the proximal regeneration of a given nerve starts after disruption, and has been utilized as control for many experimental studies ${ }^{10,11}$.

The utilization of the sensory nerve has already shown to be beneficial following prolonged denervation. When the motor nerve was connected end-to-end to the sensory nerve ("Sensory protection"), it seemed to provide trophic environment to minimize muscle atrophy until a motor donor nerve became available. Although incapable of forming excitable neuromuscular junctions, sensory nerves were found to exert powerful trophic effects on denervated muscle fibers ${ }^{12-14}$.

The impact of the sensory nerve on the muscle trophism is under investigation ${ }^{15,16}$. The end-to-side neurorraphy can be applied in those selected cases in which no donor nerve is dispensable. Experimental models should minimize the impact on the donor nerve so that they can be clinically useful. We utilized the saphenous nerve without the necessity of a graft and without disruption of its continuity. In the future, we plan to evaluate the impact of this new nerve supply in the heterotopically transplanted muscle.

\section{Conclusions}

The proposed model is simple, reproduciple. The medial gastrocnemius had the advantage of maintaining visible mass after 60 days. The sheet of aloplastic material prevent the neural contamination in the flap in end-to-side suture.

\section{References}

1. Ballance CA, Ballance HA, Stewart P. Remarks on the operative treatment of chronic palsy of peripheral origin. Br Med J. 1903 May 2;1(2209):1009-13. 
2. Harris W, Low VW. On the importance of accurate muscular analysis in lesions of the brachial plexus; and the treatment of Erb's palsy and infantile paralysis of the upper extremity by cross-union of the nerve roots. Br Med J. 1903;2:1035-8.

3. Viterbo F, Trindade JC, Hoshino K, Mazzoni A. Latero-terminal neurorraphy without removal of the epineurial sheath: experimental study in rats. Rev Paul Med. 1992 Nov-Dec; 111(6):479.

4. Lundborg G, Zhao Q, Kanje M, et al. Can sensory and motor collateral sprouting be induced from intact peripheral nerve by endto-side anastomosis? J Hand Surg. 1994 Jun;19(3):277-82.

5. Noah EM, Williams A, Fortes W, Terzis JK. A new animal model to investigate axonal sprouting after end-to-side neurorraphy. $\mathrm{J}$ Reconst Microsurg. 1997 Jul;13(5):317-25.

6. Tonken HP, Zhang F, Sudekum AE et al. Microvascular transplant of the gastrocnemius muscle in rats. Microsurgery. 1993 Oct;14(2):120-4.

7. Black KS, Hewitt CW, Aniel M et al. Neuromuscular capabilities in long-term composite tissue allografts. Transpl Proc. 1988 Apr; $20(2$ Suppl 2):269-71.

8. Bain JR, Veltri KL, Chamberlain D, Fahnestock M. Improved functional recovery of denervated skeletal muscle after temporary sensory nerve innervation. Neuroscience. 2001 Mar;103(2):503-10.

9. Spencer PS, Weinberg HJ, Raine CS, Prineas JW. The perineurial window - a new model of focal demyelination and remyelination. Brain Res. 1975 Oct;96(2):323-9.

10. Arasisis G, Mackinnon SE. End-to-side neurorraphy resulting in limited sensory regeneration in a rat model. Ann Otol Rhin Laringol. 1997 Jun;106(6):506-12.

11. Hynes NM, Bain JR, Veltri KL, Maguire JA. Preservation of denervated muscle by sensory protection in rats. J Reconstr Microsurgery. 1997 Jul;13(5):337-43.

12. Papakonstantinou KC, Kamin E, Terzis JK. Muscle preservation by prolonged sensory protection. J Reconstr Microsurgery. 2002 Apr;18(3):173-82; discussion 183-4.
13. De-Doncker, Picquet F, Falempin M. Effects of cutaneous receptor stimulation on muscular atrophy developed in hindlimb unloading condition. J Appl Physiol. 2000 Dec;89(6):2344-51.

14. Dautel G, Braga da Silva J, Floquet J, Merle M. Experimental study of the trophic effects of reinnervation of pedicled muscle flaps. Chirurgie. 1992;118(3):122-9; discussion 129-30.

15. Tiangco, D. IGF-I and end-to-side nerve repair: a dose response study. J Reconstr Microsurgery. 2001 May;17(4):247-56.

16. Jaeger $\mathrm{MR}^{1}$, Braga-Silva J, Gehlen D, Pereira-Filho Gde A, Zettler CG, de Souza MA, Veas JR, Sebben A. End-to-end versus endto-side motor and sensory neurorrhaphy in the repair of the acute muscle denervation. Ann Plast Surg. 2011 Oct; 67(4):391-6.

\section{Correspondence:}

Dr. Marcos Jaeger

Rua Attilio Bilibio, 120/106

91530-008 Porto Alegre - RS Brasil

Fax: (55 51)3028-8738

marcosjaeger@hotmail.com

${ }^{1}$ Research performed at Microsurgery Research Center, Pontific Catholic University of Rio Grande do Sul, Porto Alegre-RS, Brazil. 